Tutorial:

\title{
Comprehensive Benchmark Procedures for Commissioning, Quality Assurance and Treatment Delivery for Quality Cancer Therapy Using Co- 60 Teletherapy Machine
}

\author{
Sadiq R. Malik, Motiur Rahman, Mohsin Mia, Abdul Jobber \\ Ashish K. Bairagi, Shohel Reza, Mushfika Ahmed, and Nilufa Yeasmin \\ Division of Radiation Oncology, Delta Hospital Ltd., Mirpur -1 Dhaka-1216, Bangladesh. \\ e-mail: smailk2008@gmail.com; motiur.delta@gmail.com
}

Received: 20 September 2013, Accepted: 20 December 2013

\section{Contents:}

1. Basic Overview: An Introduction

1.1 Established Protocols

1.2 Measurement Parameters

1.3 Goal

2. Material and Methods

$2.1 \quad$ Materials

2.1.1 Description of Co-60 machine

2.1.2 Description of QA related equipment/accessories

2.2 Methods
2.2.1 Quality Assurance (QA) of Co-60 Therapy Machine
2.2.2 Annual or intermittent checks
2.2.3 Other checks and observation for the machine

3. Mechanical

3.1. Coincidence of Light Field VS Radiation Field

3.2. Alignment between opposing fields: Rotational Checks of Gantry

3.3. Accuracy of Optical Indicator

3.4. Accuracy of Gantry Angle Indicator

3.5. Accuracy of Collimator Angle Indicator

3.6. Accuracy of Field Sizes

3.7. Axis of rotation of collimator

3.8. Wedge Transmission Factor

3.9. Tray Transmission Factor

3.10. Radio isocentric size by Radiographic Image

3.11. Safety Interlock

4. Dosimetry

4.1. Verification of percentage Depth Dose (PDD)

4.2. Profile

4.3. Beam Quality or Quality index Q

4.4. Linearity Check

4.5. Absorbed Dose in Water for $\mathrm{D}_{\mathrm{W}}$

4.5.1 Pressure, temperature and humidity correction $\left(\mathrm{K}_{\mathrm{TP}}\right)$

4.5.2 Polarity effect $\left(\mathrm{K}_{\mathrm{pol}}\right)$

4.5.3 Ion Recombination $\left(\mathrm{K}_{\mathrm{s}}\right)$

4.5.4 Measurements and results

4.6. Cobalt-60 Treatment time calculation spread sheet to deliver prescription dose

4.7. Constancy Check

4.8. Output Factor Measurements

4.9. Daily Output check.

5. Discussion

6. Acknowledgment

7. Bibliography 


\section{BASIC OVERVIEW: AN INTRODUCTION}

\subsection{Established Protocols}

Assuming that a radiation Oncology Physicist is given the responsibility to use a Co-60 teletherapy machine for radiation therapy, s/he needs to evaluate its performance in order to produce a beam quality for an accurate dose delivery to the concerned anatomy. Therefore, we must comply with the established and proven protocols as well as apply all other available technologies, old and new, to quantify the beam output of the machine. To achieve this, the physicist has to perform a number of elaborate segments of measurements as outlined in this report. Recommendations of international Task Groups are matched and incorporated in the following measurements before accepting, commissioning and delivery of radiation therapy treatments to cancer patients.

\subsection{Measurement Parameters}

Mechanical parameters that relate to Gantry, Couch, Collimators and Field Sizes are expressed in millimeters $(\mathrm{mm})$ and degrees $\left(\theta^{0}\right)$ with regards to the appropriate measurement parameters of the components of the machine. Besides, Dosimetric Properties viz. beam profiles, penumbra, PDD (Percent Depth Dose), dose calculations in tissue and treatment delivery encompass various types of measurements in order to conform and quantify with international Task Group recommendations viz. TRS-398 and TG-40 (1994b, 2005) It is imperative that the systematic guidelines enunciated here should be established to assure a quality cancer treatment.

Implementations of quality control on machine, using the protocolsTRS-398 and TG-40 prior to the delivery of treatment is a necessity. The implications of such a report, replicating the benchmark guidelines, are enunciated here. Cancer Therapy using Co-60 teletherapy machines, in Bangladesh as well as in other countries, may benefit from the systematic presentations of procedures, tables, figures and measurements as documented in this tutorial. Limits of tolerance in mechanical and dose values, with beam check parameters, are evaluated in this paper. The teletherapy machine is then accepted and commissioned to deliver the prescribed dose to the disease of cancer patients.

\subsection{Goal}

A cobalt therapy machine uses a radionuclide produced in a Nuclear Reactor having a mean $\gamma$-energy of $1.25 \mathrm{MeV}$. Energy of such photons has a skin sparing property as maximum dose is achieved at 5 $\mathrm{mm}$ depth below a tissue (i.e. skin) or any equivalent material. The activity of the source decays by about $1.09 \%$ per month as the half-life of Co-60 is 5.26 years (Metcalfe Peter and Kron 1997). The penumbra is larger, compared to that of Linear Accelerators, as the source diameter is about $2 \mathrm{~cm}$ (Cunningham 1983). A short source to surface distance (SSD) of $80 \mathrm{~cm}$ is used to achieve a reasonable dose rate for treatments, whether palliative or curative.

The series of measurements presented here, to act as a guide to the use of such a machine for cancer therapy, have been aimed at the verification of all machine and radiation characteristics in order to ensure quality cancer therapy.

\section{MATERIALS AND METHODS}

\subsection{Materials}

In the present study, essential measurements for commissioning and acceptance has been carried out following the guidelines, policy and procedures of TG-40 and TRS-398 (1994a, 2005). Table 1 summarizes most of the essential values, with tolerance levels, in the evaluation of the machine which should be established by appropriate procedures of measurement. The objective of this work may, therefore, be taken as a reference for any user or, for that matter, the Nuclear Regulatory Authority (NRA) for the verification of quality performance of the machine, while using it at any Cancer Treatment Center (CTC). Detailed information on materials and methods are compiled here. The 
following description pertains to the facilities available at the Radiation Oncology Division, Delta Hospital Ltd., Dhaka, Bangladesh. The procedures cited here can serve as examples for newcomers into this field.

\subsubsection{Desription of the Co-60 Teletherapy Machines}

Our hospital, Delta Hospital Ltd, has two $\mathrm{Co}^{60}$ teletherapy machines providing external beams of photons for cancer therapy. Let the names of the two machines be Cobalt-I and Cobalt-II. These two teletherapy sources differ in their activities and hence the dose rates. The dose rate is an essential parameter in the calculation of prescribed dose. The salient features of these two Cobalt sources are as follows:

\section{Cobalt-I}

Manufacturer : G.E Medical System, USA

Model : ALCYON II P

Date of installation : June, 1994

1st time source change date: March 2005

2nd time source change date: June 2013

Present activity of the source: $6974 \mathrm{Ci}$

\section{Cobalt-II}

Manufacturer : Cis Bio. Int. France.

Model : CIRUS

Date of Installation : February, 2002.

1st time source change date: June 2013.

Present activity of the source: $\mathbf{8 2 4 0 C i}$

\subsubsection{Description of $Q A$ related equipment/accessories}

Here at Delta Hospital Ltd, we are using an IBA Farmer type ion chamber FC-65, a WP1D Phantom and Electrometer Dose-1 for absolute dosimetrey. The water phantom and ion chamber are also used to scan the beam of radiation in $2 \mathrm{D}$. We also use a small water phantom and solid water phantoms (slabs) together with ion chambers, FC-65, for weekly and monthly check up of dose variations of the photon beams from $\mathrm{Co}^{60}$. We use Barometer and Thermometer GTD 1100 from GREISINGER electronics, Germany, for pressure and temperature measurements. The temperature and pressure readings are required for dosimetry purposes.

\subsection{Methods: Quality Assurance (QA) of Co-60 Therapy Machine}

The methods for different tests and checks are briefly described in the following sections where the section titles describe the checks and the parameters used in our typical tests. The tables within each section show the outputs that our measurements produced, which can be used as a guide. The tolerance values are also indicated. QA is a routine process and the QA related parameters will influence the functional performance and dosimetric accuracy of the applied dose to the patients. These measurements are essential in supporting preventive maintenance and delivery of the prescribed dose $(\boldsymbol{R} \boldsymbol{x})$ to patients.

\subsubsection{Annual or intermittent checks}

Annual or intermittent measurements are made to evaluate the performance of the therapy machine for which tolerance values from Task Groups are given in Table-1 (1994a, 2005). 


\subsubsection{Other Checks and Observations for the machine}

We performed the following checks to ascertain the functional integrity of the machine where the cobalt source is driven pneumatically for patient exposure.

a. On - Off Procedure : OK

b. Exposure On (Start) and Off (end) Time $=T_{2}-T_{1}=\mathbf{3}$ units, where $T_{2}$ and $T_{1}$ stand for exit and entry times of the Cobalt source. The time unit is defined as, 100 units $=1$ minute. We used $\mathrm{T}_{2}$ $=103$ units and $\mathrm{T}_{1}=100$ units.

If such procedures are followed, delivery of the prescribed dose to the patients will be accurate.

Table-1: Functional Parameters for a $\mathbf{C o}^{\mathbf{6 0}}$ Teletherapy Machine

\begin{tabular}{|c|c|c|c|c|}
\hline $\mathrm{S} / \mathrm{L}$ & Procedure & $\begin{array}{l}\text { Measured } \\
\text { Value/finding }\end{array}$ & $\begin{array}{l}\text { Tolerance } \\
\text { Value/finding }\end{array}$ & $\begin{array}{l}\text { Remarks/ } \\
\text { Limitations }\end{array}$ \\
\hline 1. & Operational Parameter & Functional & Functional & \\
\hline 2. & Door Interlock & Functional & Functional & \\
\hline 3. & Emergency off & Functional & Functional & \\
\hline 4. & Radiation room monitor & Functional & Functional & \\
\hline 5. & Audiovisual Monitor & Functional & Functional & \\
\hline 6. & Lasers & $1 \mathrm{~mm}$ & $2 \mathrm{~mm}$ & \\
\hline 7. & Distance Indicator ODI) & $1 \mathrm{~mm}$ & $2 \mathrm{~mm}$ & $\mathrm{SSD}=80.1 \mathrm{~cm}$ \\
\hline 8. & Light/radiation field coincidence & $1 \mathrm{~mm}$ & $2 \mathrm{~mm}$ & \\
\hline 9. & $\begin{array}{l}\text { Coincidence of Gantry, } \\
\text { Collimator \& Table axis with } \\
\text { isocentric rotation }\end{array}$ & $2 \mathrm{~mm}$ & $2 \mathrm{~mm}$ dia & \\
\hline 10. & $\begin{array}{l}\text { Radiation and Mechanical } \\
\text { isocenter coincidence }\end{array}$ & $2 \mathrm{~mm}$ & $2 \mathrm{~mm}$ dia & \\
\hline 11. & Opposing field alignment & Aligned & Aligned & \\
\hline 12. & Table Vertical Movement & $1 \mathrm{~mm}$ & $2 \mathrm{~mm}$ & $\mathrm{SS}=70-100 \mathrm{~cm}$ \\
\hline 13. & Table Segment & $2 \mathrm{~mm}$ & $2 \mathrm{~mm}$ & \\
\hline \multirow[t]{2}{*}{14.} & \multirow[t]{2}{*}{ Field Size Indicator } & \multirow[t]{2}{*}{$\begin{array}{l}2 \mathrm{~mm}(5 \times 5- \\
25 \times 25)\end{array}$} & \multirow[t]{2}{*}{$2 \mathrm{~mm}$} & $\begin{array}{l}\mathrm{AP}(\mathrm{x}= \pm 2 \mathrm{~mm}, \mathrm{y} \pm 2 \mathrm{~m} \\
\mathrm{m})\end{array}$ \\
\hline & & & & $\begin{array}{l}\operatorname{Lat}(\mathrm{x} \pm 5 \mathrm{~mm}, \mathrm{y} \pm 5 \mathrm{~m} \\
\mathrm{m})\end{array}$ \\
\hline 15. & $\begin{array}{l}\text { Gantry \& collimator Angle } \\
\text { Indicator }\end{array}$ & $<1^{\circ}$ & $1^{\circ}$ & \\
\hline 16. & Field light Intensity & Functional & Functional & \\
\hline 17. & $\begin{array}{l}\text { Timer Linearity \& Consistency } \\
\text { \& Error }\end{array}$ & $1 \%$ & $1 \%$ & \\
\hline 18. & Central Axis PDD & $2 \%$ & $2 \%$ & \\
\hline 19. & $\begin{array}{ll}\text { Flatness, } & \text { Symmetry, } \\
\text { Penumbra }\left(\mathrm{Co}^{60}\right) & \end{array}$ & 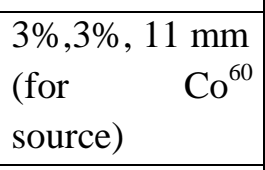 & $3 \%, 3 \%$ & \\
\hline 20. & Absolute Dose Rate Consistency & $1 \%$ & $2 \%$ & \\
\hline
\end{tabular}


Shielding blocks of various geometrical shapes, may be required for treatment, to define the treatment field for a patient. A common material used is Cerrobend, also known as Lipowitz metal, and consists of $50 \%$ Bismuth, $26.7 \%$ lead, $13.3 \%$ tin and $10.0 \%$ Cadmium. Cerrobend has a density of $9.4 \mathrm{~g} / \mathrm{cm}^{3}$ (i.e., $83 \%$ of lead density) and melts at $70^{\circ} \mathrm{C}$. This shield is approximately $6 \mathrm{~cm}$ thick so as to allow $\leq$ $5 \%$ primary transmission.

\section{MECHANICAL}

\subsection{Coincidence of Light Field Vs Radiation Field (1994b)}

To verify this parameter, a CR Film was placed at isocenter with $10 \times 10 \mathrm{~cm}^{2}$ field size. The corner of the light field and the position of the cross hairs have been marked by lead markers or by pins. The film was exposed to about $1.9 \mathrm{sec}$ for the activity (6974Ci in our Cobalt I) of this source. The agreement of light field and radiation fields is, within $\mathbf{\pm 2} \mathbf{~} \mathbf{m m}$ as shown in Figure 1 .

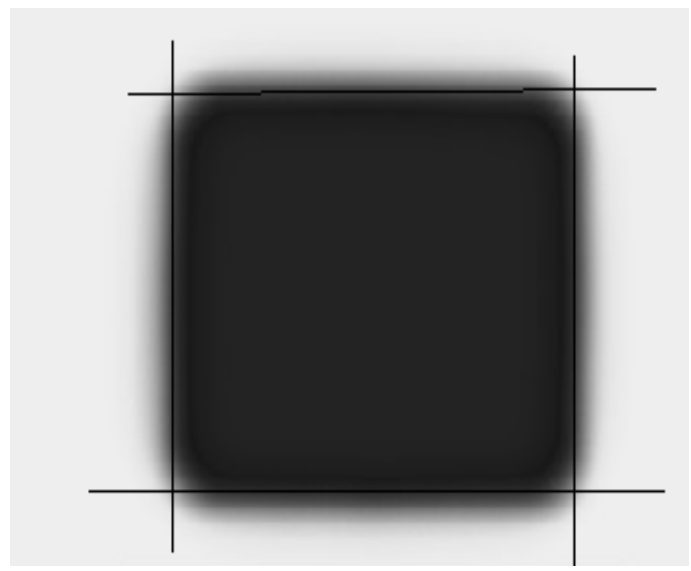

Fig 1: Picture of Radiation field against Light field. Field Size 10x10. Film has been exposed $\geq$ for $1.9 \mathrm{sec}$ for $6974 \mathrm{Ci}$ (CIRUS)
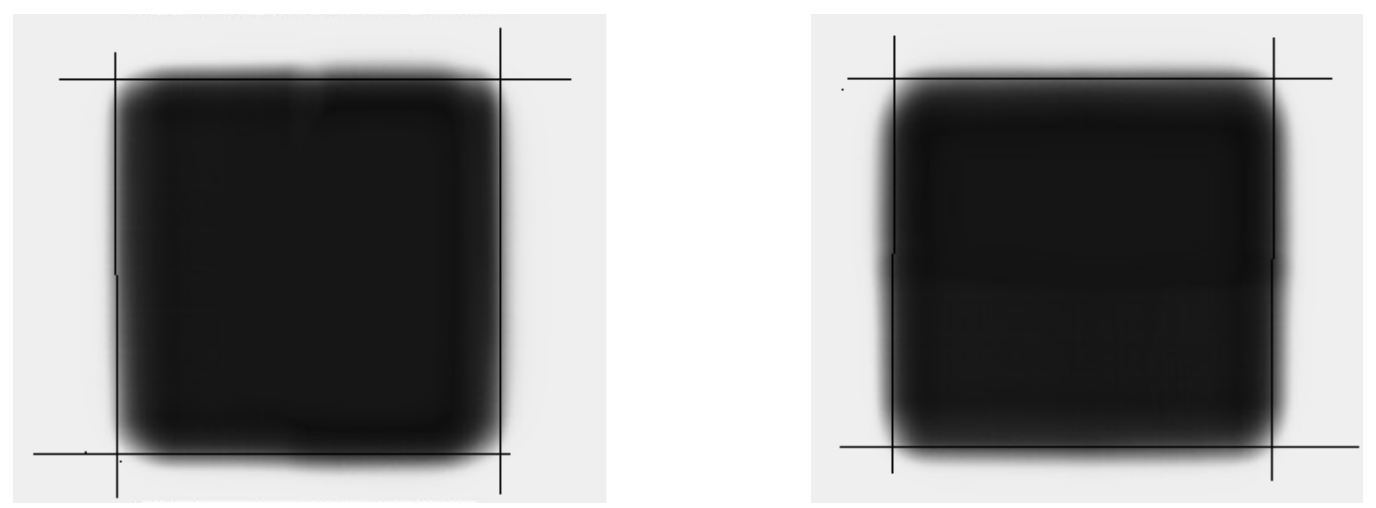

Fig. 2: Gantry Rotation for alignment check of opposing fields with half mask. For Gantry at $0^{\circ} \& 180^{\circ}$ (left); for Gantry at $90^{\circ} \& 270^{\circ}$ (right)

\subsection{Alignment between opposing fields: Rotational Checks of Gantry}


To verify this parameter, a CR Film was placed on the smooth and flat surface of the couch at the isocenter. Field size of $10 \times 10 \mathrm{~cm}^{2}$ was obtained by moving the collimator. Half of the field size was blocked by lead shields at Gantry $\left(\theta^{0}=0\right)$ and, after exposure, the Gantry position was rotated by $180^{\circ}$ for another exposure. The light field and the position of the cross hair were marked using lead $(\mathrm{Pb})$ shots. The film was exposed for about $1.9 \mathrm{sec}$. The light and radiation fields should agree to within $\mathbf{\pm 2}$ $\mathbf{m m}$, which was obtained in our case (Figures 2). This is within the acceptable tolerance value for this measurement (Ref: Table 1). We can also check the lateral alignment for $90^{\circ}$ and $270^{\circ}$ following similar procedures. We recommend any user of Cobalt Machine to perform this measurement.

\subsection{Accuracy of Optical Indicator}

The distance was measured with the 'front pointer' and compared with that of an 'Optical Distance Indicator' (ODI). The results are given in Table 2, which relates to the accuracy of such a measurement (Khan 1984a). Tolerance value is $\mathbf{\pm 2} \mathbf{~ m m}$ (Ref: Table 1).

Table 2: Accuracy of Optical Indicator

\begin{tabular}{|l|l|l|}
\hline SSD Indicated by pointer $(\mathrm{cm})$ & SSD Measured with ODI $(\mathrm{cm})$ & Deviation $(\mathrm{mm})$ \\
\hline 80 & 79.8 & 2 \\
\hline 100 & 100 & 0 \\
\hline 110 & 110.2 & 2 \\
\hline 120 & 120.1 & 1 \\
\hline
\end{tabular}

\subsection{Accuracy of Gantry Angle Indicator (1994b, 2004)}

Gantry Angles at $0^{\circ}, 45^{\circ}, 90^{\circ}, 180^{\circ}$ and $270^{\circ}$ were determined using a sprit level. The accuracy of the digital readout was checked against the mechanical readout. The results are given in Table 3 . Tolerance value is $\boldsymbol{\theta} \pm \mathbf{1}^{\mathbf{0}}$ (Ref: Table 1$)$.

Table 3: Accuracy of Gantry Angle when rotated at various angles

\begin{tabular}{|l|l|l|l|}
\hline Sprit Level & Digital Readout (Deg.) & $\begin{array}{l}\text { Mechanical Readout } \\
(\text { Deg. })\end{array}$ & Deviation (Deg.) \\
\hline $0^{0}$ & $0^{0}$ & $0^{0}$ & $0^{0}$ \\
\hline $45^{0}$ & $44.7^{0}$ & $45^{0}$ & $0.3^{0}$ \\
\hline $90^{0}$ & $90.5^{0}$ & $90^{0}$ & $0.5^{0}$ \\
\hline $180^{0}$ & 179 & $180^{0}$ & $1^{0}$ \\
\hline $270^{0}$ & $269.8^{0}$ & 270 & $0.2^{0}$ \\
\hline
\end{tabular}

\subsection{Accuracy of Collimator Angle Indicator (1994b, 2004)}

With the Gantry Angle at $90^{\circ}$, a spirit level was placed on the Collimator Housing to check the accuracy of the collimator angle indicators, both digital and mechanical. The results are given in Table 4. Tolerance value is $\boldsymbol{\theta} \pm \mathbf{1}^{\mathbf{0}}$ ( (see table 1 ).

Table 4: Accuracy of collimator angle by Digital and Mechanical readout

\begin{tabular}{|l|l|l|l|}
\hline Spirit Level (Deg.) & Digital Readout(Deg.) & Mechanical readout (Deg.) & Deviation (Deg.) \\
\hline $0^{0}$ & $0^{0}$ & $0^{0}$ & $0^{0}$ \\
\hline $45^{0}$ & $45^{0}$ & $44.8^{0}$ & $0.2^{0}$ \\
\hline $90^{0}$ & $90^{0}$ & $89.7^{0}$ & $0.3^{0}$ \\
\hline
\end{tabular}




\begin{tabular}{|l|l|l|l|}
\hline $270^{\circ}$ & $270^{\circ}$ & $269.8^{0}$ & $0.2^{0}$ \\
\hline
\end{tabular}

3.6 Accuracy of Field Sizes (1994b, 2004)

A millimeter graph paper was utilized to measure the field size accuracy. After setting the jaw for $\mathrm{X}$ and $\mathrm{Y}$ the field light was checked at $80 \mathrm{~cm}$, the Source to Surface Distance (SSD) for such a measurement (set at Isocenter, $80 \mathrm{~cm}$ ). The graph paper was placed on the COUCH at $80 \mathrm{~cm}$. Jaws of the collimator were adjusted to vary the Field Sizes and the corresponding accuracy of the Field Sizes were checked. The results are given in Table 5. Tolerance value or deviation of such measured values of the Field Sizes should be within $\pm 2 \mathrm{~mm}$ (see table 1).

Table 5: Test for accuracy of Field Sizes

\begin{tabular}{|l|l|l|l|l|}
\hline $\begin{array}{l}\text { Dial Setting } \\
\text { Field size } \\
(\mathrm{cm} \times \mathrm{cm})\end{array}$ & $\begin{array}{l}\text { Measured Light } \\
\text { Field X (for } \\
\text { lower jaw }) \\
(\mathrm{cm})\end{array}$ & $\begin{array}{l}\text { Measured Light } \\
\text { Field Y (for upper } \\
\text { jaw) } \\
(\mathrm{cm})\end{array}$ & $\begin{array}{l}\text { Deviation of } \\
\text { Field Size X } \\
\text { (for lower Jaw) } \\
(\mathrm{cm})\end{array}$ & $\begin{array}{l}\text { Deviation of } \\
\text { Field Size Y } \\
(\text { for upper Jaw) } \\
(\mathrm{cm})\end{array}$ \\
\hline $4 \times 4$ & 3.8 & 3.8 & 0.2 & 0.2 \\
\hline $5 \times 5$ & 4.9 & 4.9 & 0.1 & 0.1 \\
\hline $10 \times 10$ & 9.9 & 10 & 0.1 & 0.0 \\
\hline $15 \times 15$ & 14.9 & 15 & 0.1 & 0.0 \\
\hline $20 \times 20$ & 19.9 & 20 & 0.1 & 0.0 \\
\hline $25 \times 25$ & 24.9 & 24.9 & 0.1 & 0.1 \\
\hline $30 \times 30$ & 29.9 & 29.9 & 0.1 & 0.1 \\
\hline
\end{tabular}

\subsection{Axis of rotation of collimator}

A mechanical study for the axis of rotation of the collimator at gantry angle $\theta$ was observed by defining the geometric center of the locus of points generated by the light field cross-hair indication at $80 \mathrm{~cm}$, when the collimator is rotated through its full rotational range.

\subsection{Wedge Transmission Factor}

Wedge Factor has been measured in water, at a depth of $5 \mathrm{~cm}$, using SSD of $80 \mathrm{~cm}$ and a field size of $10 x 10 \mathrm{~cm}$. An IBA Farmer Type ionization chamber was used for the measurements. The following parameters were used in acquiring the data as shown in Table 6 (Khan 1984a).

Energy: Cobalt-60. Field Size $=10 \times 10 \mathrm{~cm}$ at $\mathrm{SSD}=80 \mathrm{~cm}$. Ion Chamber was placed at a depth of 5 $\mathrm{cm}$ in water. Beam ON Time was fixed at $=1$ minute for each of the measurements.

Wedge Factor is the ratio of readings of the electrometer in Coulomb for the wedged field to that for the open field (no wedge).

Open Field reading: $3.796 \times 10^{-8} \mathrm{C}$ for the activity of $6974.35 \mathrm{C} i$ source of this teletherapy machine. Measured Wedge Factor should be within $\pm 2 \%$ of the manufacturer's value. 
Table 6: Wedge Transmission Factor

\begin{tabular}{|c|c|c|c|c|c|c|}
\hline $\operatorname{Angle}(\theta)$ & $\begin{array}{l}\text { Wedge } \\
\text { Size }(\mathrm{cm})\end{array}$ & $\mathrm{M} 1 \ldots \mathrm{X} 10^{-8} \mathrm{C}$ & $\mathrm{M} 2 \ldots \mathrm{X} 10^{-8} \mathrm{C}$ & Average & Factor & $\begin{array}{l}\text { Manufacturer's } \\
\text { value }\end{array}$ \\
\hline 15 & $8 \times 15$ & 3.149 & 3.149 & 3.149 & 0.839 & 0.835 \\
\hline 30 & $10 \times 15$ & 2.589 & 2.589 & 2.589 & 0.682 & 0.682 \\
\hline 45 & $10 \times 15$ & 2.107 & 2.107 & 2.107 & 0.555 & 0.553 \\
\hline 60 & $10 \times 15$ & 1.468 & 1.468 & 1.468 & 0.368 & 0.367 \\
\hline
\end{tabular}

\subsection{Tray transmission factor}

Tray transmission factor was measured at $5 \mathrm{~cm}$ depth in water for $80 \mathrm{~cm} \mathrm{SSD} \mathrm{and} 10 \mathrm{x} 10 \mathrm{~cm}$ field using IBA Farmer type ionization chamber (Khan 1984a).The following parameters were used. Measured Transmission Factor should be within $\pm 2 \%$ of the manufacturer's value.

Energy: Cobalt-60, F.S: $10 x 10 \mathrm{~cm}$ for $\mathrm{SSD}=80 \mathrm{~cm}$. Ionization Chamber was placed at a depth $=5$ $\mathrm{cm}$ of water. Beam on Time $=1$ minute

Table 7: Tray Transmission Factor

\begin{tabular}{|l|l|l|l|}
\hline Open Field Reading & $\begin{array}{l}\text { Reading with } \\
\text { Tray }\end{array}$ & $\begin{array}{l}\text { Tray Transmission } \\
\text { Factor }\end{array}$ & $\begin{array}{l}\text { Manufacturer's } \\
\text { value }\end{array}$ \\
\hline $3.506 \times 10^{-8} \mathrm{C}$ & $3.373 \times 10^{-8} \mathrm{C}$ & 0.962 & 0.96 \\
\hline
\end{tabular}

3.10 Radiation isocentric size by Radiographic Image: (Star Shot) (2004)

Radiation isocenter size has been evaluated and confirmed radiographically. Figures 3, 4 and 5 depict STAR FILM shots for Gantry, Collimator and Couch to a tolerance of $\pm 1.0 \mathrm{~mm}$. Figures for the following components of the machine are listed below.

$\begin{array}{ll}\text { i. } & \text { Collimator Rotation } \\ \text { ii. } & \text { Gantry Angles } \\ \text { iii. } & \text { Couch Position }\end{array}$

\section{i: Star Shot for Collimator angle $(C<\theta)$}

Procedure: CR film has been placed at $80 \mathrm{SSD}$ for exposures at Collimator angles of $0^{\circ}, 45^{\circ}, 90^{\circ}$, $135^{\circ}$. Exposures have been carried out for $1.9 \mathrm{sec}$ at the centre of film using the isocentric rotation of the collimator. The film has been placed on the top of couch at a Field Size of $4 \mathrm{~cm} \times 30 \mathrm{~cm}$.

\section{ii: Star Shot for Gantry Angle (G> $)$}

Procedure: CR film has been placed at 80 SSD for exposures at Gantry angles $0^{\circ}, \mathbf{4 5}^{\circ}, \mathbf{9 0}^{\circ}, \mathbf{1 3 5}^{\circ}$. An exposure has been carried out for $1.9 \mathrm{sec}$ at the centre of film with isocentric rotation of gantry. The film has been placed perpendicularly to the Gantry at a Field Size of $4 \mathrm{~cm} \times 30 \mathrm{~cm}$ in the plane of the rotation of the gantry.

\section{iii: Star shot Couch angle $(\mathrm{C}<\boldsymbol{\theta})$}

Procedure: CR film has been placed at $80 \mathrm{~cm}$ (SSD) with exposures at Couch angles $\mathbf{0}^{\mathbf{0}}, \mathbf{4 5}^{\mathbf{0}}, \mathbf{9 0}^{\mathbf{0}}$, $\mathbf{1 3 5}^{\circ}$. Exposures have been carried out for $1.9 \mathrm{sec}$, at the centre of film, with isocentric rotation of the couch. Film has been placed on the top of couch with a Field Size $=4 \mathrm{~cm} \times 30 \mathrm{~cm}$. Figure 5 shows the Couch Star Shot as observed on the film. 


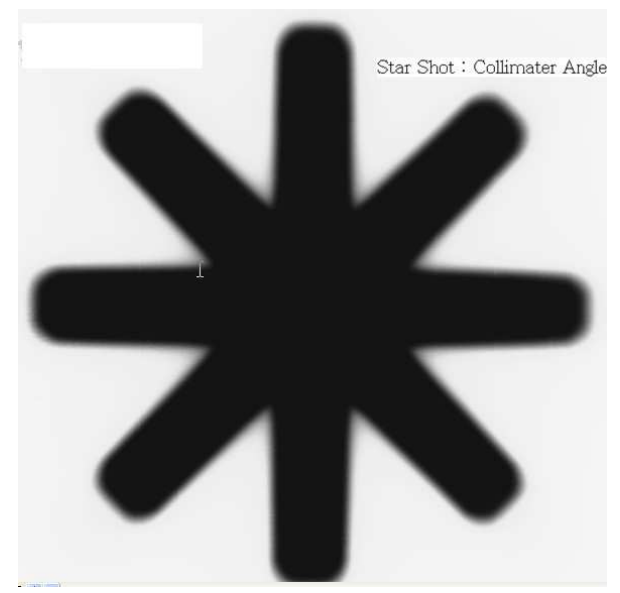

Fig 3: Star shot for collimator at various angles $(\mathbf{C}<\boldsymbol{\theta})$

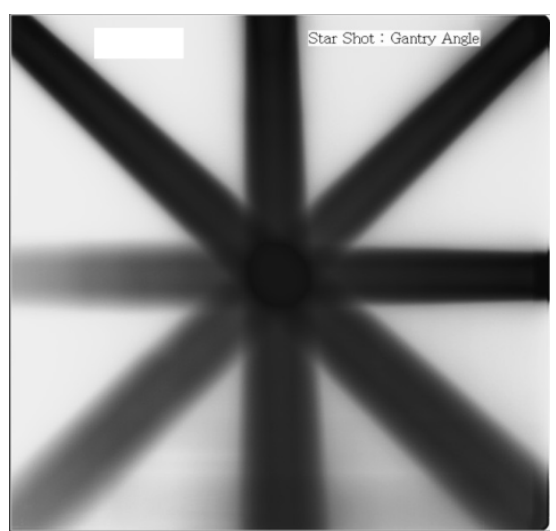

Fig 4: Gantry Star Shot, $\mathrm{G}>\theta$

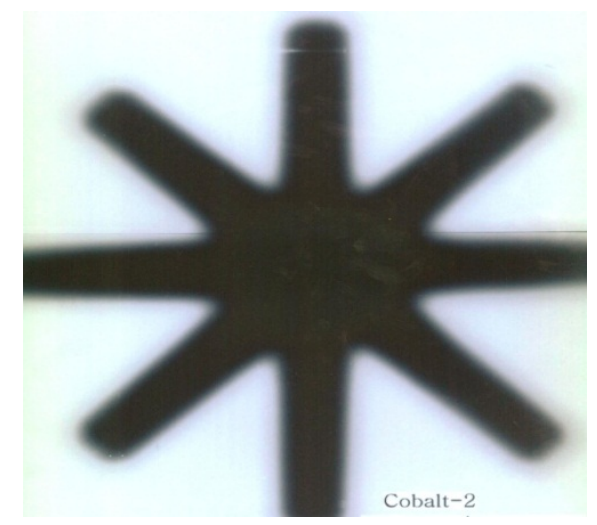

Fig 5: Couch Star Shot

\subsection{Safety Interlock (2004)}

\section{a. Indicator or Warning Lights}

The "Radiation On" indicator lights beside the door and in the Treatment

Room has been checked and observed to function properly.

\section{b. Audio/Video Monitor}

Room Audio System which included Video Monitor has been working properly.

\section{c. Door Interlock}

The door interlock terminates the beam when the door is opened. Exposure and beam cannot be turned on when for an open door.

\section{d. Emergency Buttons}

The Emergency Button shut off the machine, when it was activated.

\section{DOSIMETRY}

4.1 Verification of Percentage Depth Dose (PDD) (1990, Allen and Blagojevic 1996)

Scan of percentage depth dose for field sizes from $4 x 4$ to $32 x 32 \mathrm{~cm}^{2}$ has been obtained using RFA300 Phantom (IBA). PDD's for the measured $10 \times 10 \mathrm{~cm}^{2}$ field are shown for the indicated depths at $\mathrm{SSD}=80 \mathrm{~cm}$. PDD has been acquired from the scanning data of 3D Phantom RFA300 for $10 \times 10 \mathrm{~cm}^{2}$ field sizes, at different depths, as given in Fig 6. The PDD values have also been acquired at different 
depths of water phantom using the ion chamber FC-65 which is tabulated in Table 8. The PDD values in both of these measurements agree with each other, as shown

Table 8: Depth vs. PDD dose

\begin{tabular}{|l|l|l|}
\hline Depth ( cm) & $\begin{array}{l}\text { Reading } \\
\text { Coulomb (C) }\end{array}$ & $\begin{array}{l}\text { PDD } \\
(\%)\end{array}$ \\
\hline 0.5 & $4.450 \times 10^{-8}$ & 100 \\
\hline 1 & $4.409 \times 10^{-8}$ & 99.1 \\
\hline 3 & $4.005 \times 10^{-8}$ & 90 \\
\hline 5 & $3.506 \times 10^{-8}$ & 78.78 \\
\hline 10 & $2.528 \times 10^{-8}$ & 56.6 \\
\hline 15 & $1.726 \times 10^{-8}$ & 38.8 \\
\hline 20 & $1.183 \times 10^{-8}$ & 26.6 \\
\hline
\end{tabular}

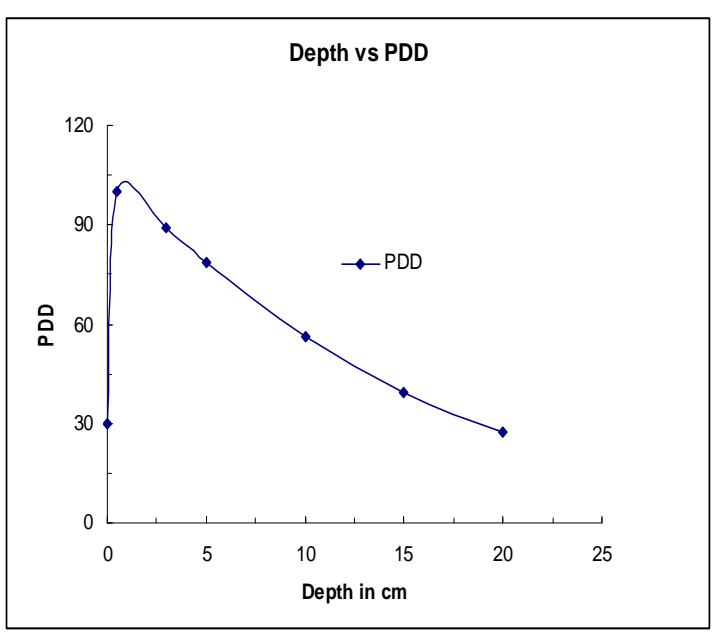

Fig 6: Percentage Depth dose

\subsection{Profile (2005)}

Scanning of abeam provides the lateral profile which offers a check for the flatness and Symmetry of the beam. Table 9, shown below, shows the acquired data of the scans to provide the Symmetry \& Flatness criteria of the beam. Flatness and Symmetry should vary within $\mathbf{\pm 3 \%}$ from mid line of the profile graph (fig 7).

Table 9: Distance vs. Dose (Profile)

\begin{tabular}{|l|l|l|l|}
\hline Coordinate & $\begin{array}{l}\text { Distance from } \\
\text { centre }(\mathrm{cm})\end{array}$ & $\begin{array}{l}\text { M. Readings } \\
(\mathrm{C})\end{array}$ & Dose (\%) \\
\hline \multirow{5}{*}{$\mathrm{X}$} & 8 & 0.162678 & 4.64 \\
\cline { 2 - 4 } & 6 & 0.5767 & 16.45 \\
\cline { 2 - 4 } & 5.5 & 1.2299 & 35.08 \\
\cline { 2 - 4 } & 5 & 2.0951 & 59.76 \\
\cline { 2 - 4 } & 4.5 & 3.0060 & 85.74 \\
\cline { 2 - 4 } & 4 & 3.3250 & 94.84 \\
\hline \multirow{5}{*}{$\mathrm{X}^{\prime}$} & 3 & 3.4414 & 98.16 \\
\hline & 2 & 3.4754 & 99.13 \\
\hline & 0 & 3.5060 & 100 \\
\hline & -2 & 3.4783 & 99.21 \\
\cline { 2 - 5 } & -3 & 3.4414 & 98.16 \\
\cline { 2 - 4 } & -4 & 3.3619 & 95.89 \\
\cline { 2 - 4 } & -4.5 & 3.0796 & 87.84 \\
\cline { 2 - 4 } & -5 & 2.4047 & 68.59 \\
\cline { 2 - 4 } & -5.5 & 1.4753 & 42.08 \\
\hline & -6 & 0.7608 & 21.7 \\
\hline & -8 & 0.1626 & 4.64 \\
\hline
\end{tabular}


Procedure of evaluation is cited as follows: Comparison of any arbitrary point for evaluation of Flatness and Symmetry which should be equidistant from central axis of the beam. Measurement of Cross Plan Profile (X-axis) has been done with the following set up, viz. SSD $=80 \mathrm{~cm}$, Field size $=$ $10 \times 10 \mathrm{~cm}^{2}$ for a depth of measurement, $d=d_{\text {ref }}(5$ or $10 \mathrm{~cm})$. Ion Chamber has been placed in water at a depth of $5 \mathrm{~cm}$.

Equipment: Phantom, Ion Chamber FC-65 P, Electrometer. Set up of the water phantom has been described in the previous measurement.

4.3 Beam Quality or Quality index Q: $\frac{d_{20}}{d_{10}}=\mathbf{0 . 4 6 8}$

Where $\mathbf{d}_{20}=$ Measured Charge (Coulomb) at $20 \mathrm{~cm}$ depth. $\quad \mathbf{d}_{\mathbf{1 0}}=$ Measured Charge (Coulomb) at $10 \mathrm{~cm}$ depth. Data from measurements, as obtained in table 8 , provide the Beam Quality of $\mathbf{0 . 4 6 9}$, a deviation of < $\mathbf{1 \%}$, where the acceptable limit is $\leq \mathbf{2 \%}$.

\subsection{Linearity Check}

The linearity check, time vs reading, has been carried out where the output data of the machine is tabulated. We are required to setup the water phantom at $80 \mathbf{c m}(S S D)$, where the Field Size $=10 \times 10 \mathrm{~cm}^{2}$ at a measurement depth of $5 \mathrm{~cm}$ for the position of the ion chamber. First we measure the output for a time of 0.50 minute followed by an increment, at intervals, of 0.50 minute. The reading of this output data has been plotted as shown in figure 8 . The graph should be a linear one which will pass through the origin. After the

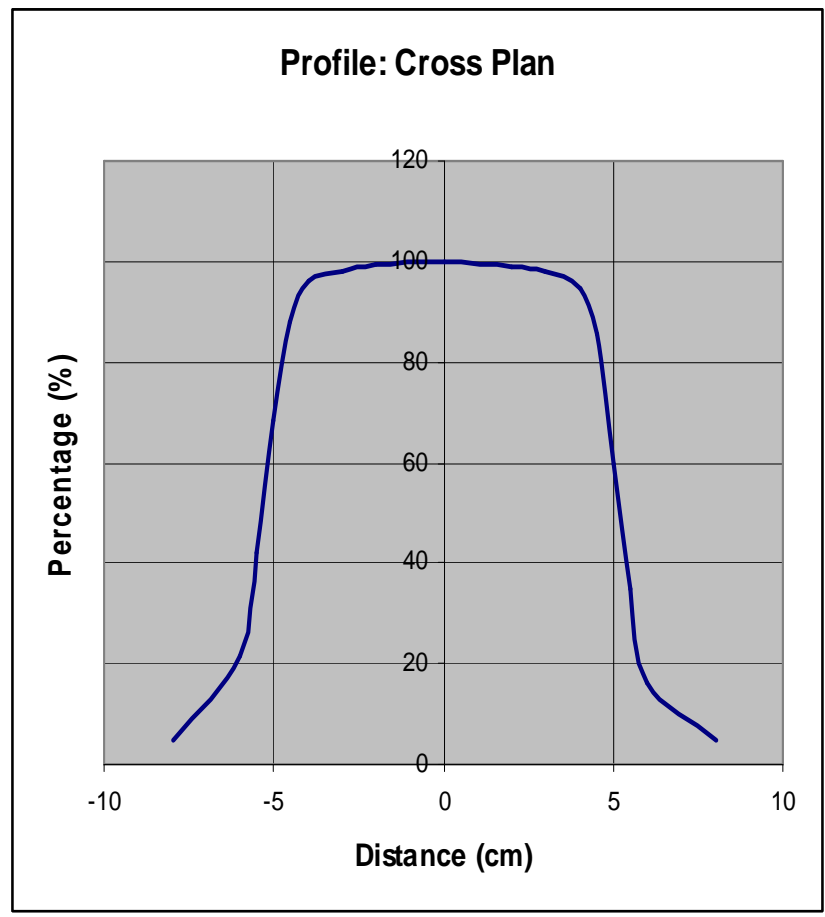

Fig7: Cross Plane Profile completion of all the mechanical and basic beam parameter checks, the linearity check will confirm the functional integrity of the dose delivery by this teletherapy machine. Dose is calculated in terms of

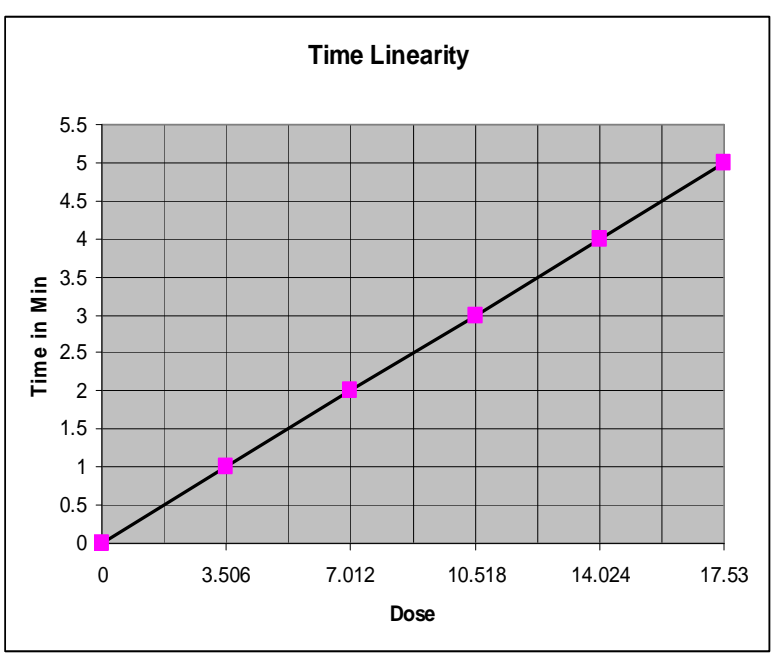

Fig 8: Time vs. Reading time to deliver a treatment. The graph should pass through origin (0.0) and hence may be presented in a format as shown in figure 8.The linearity in time and dose is required for the delivery of prescription dose (Rx) to the patient. Quality Assurance of this study is essential in the therapeutic use of the machine. Table 10 shows the measurement data and the linearity graph as figure 8 .

\subsection{Absorbed Dose in Water for $D_{w}$}

The Output of a 10x10 $\mathrm{cm}^{2}$ field was measured in water Phantom at $80 \mathrm{SSD}$ at a depth of $5.0 \mathrm{~cm}$ $\left(\mathrm{D}_{\text {ref }}\right)$, for Gamma rays, following the TRS-398 protocol (2005). The dosimetry system used 
consisted of a water proof IBA FC65P ion chamber, S/N: 1787 and IBA Dose-1 Electrometer S/N: 13527.

The formula for measuring the absorbed dose at $\mathrm{D}_{\mathrm{w}}$ is:

$$
D_{w}\left(D_{r e f}\right)=\frac{M_{1} \times K_{T P} \times K_{e l e} \times K_{P o l} \times K_{Q} \times N_{D W} \times 100}{P D D @ D_{r e f}}------(1)
$$

where,

$\mathrm{M}_{1}=$ Measured Reading at $\mathrm{D}_{\mathrm{ref}}$

$\mathrm{K}_{\mathrm{TP}}=$ Temperature and pressure correction factor

$\mathrm{K}_{\text {ele }}=$ Electrometer reading correction factor

$\mathrm{K}_{\mathrm{POL}}=$ Polarization correction factor

$\mathrm{K}_{\text {ion }}=$ Ion recombination Correction factor

$\mathrm{K}_{\mathrm{Q}}=$ Quality conversion Factor

$\mathrm{N}_{\mathrm{DW}}=$ Chamber (FC-65) calibration factor

$\mathrm{PDD}=$ Percentage Depth Dose

$D_{\text {ref }}=$ Depth of measurement

$\mathrm{D}_{\mathrm{w}}=$ Absorbed does rate to water at the reference depth $\mathrm{D}_{\text {ref }}$.

Before starting the measurements, the stability of the dosimeter system should be verified using a check source CS-137. Reasonable time should be allowed for the dosimeter to reach thermal equilibrium. For this, the Electrometer is to be switched on for about 2 hours before starting the measurement. It is always advisable to irradiate the ionization chamber with at least 500cGy, for a consistent and reproducible data.

The leakage current should always be measured before and after irradiation and ensured that it is small, typically less than about $0.1 \%$, of the current obtained during irradiation.

The calibration factor for an ionization chamber is valid only for the reference condition which applies to the calibration. Any departure from the reference condition, when using the ionization chamber in the user beam, should be corrected for the appropriate factors.

\subsubsection{Pressure, temperature and humidity correction factor( $\left.K_{\mathrm{TP}}\right)$}

The correction factor $\mathrm{K}_{\mathrm{TP}}$ is given by,

$$
K_{T P}=\frac{(273.2+T)}{\left(273.2+T_{0}\right)} \times \frac{p_{0}}{p}------(2)
$$

Where, $\mathrm{P}$ and $\mathrm{T}$ are the cavity air pressure and temperature at the time of measurements andP $\mathrm{P}_{0}$ and $\mathrm{T}_{0}$ are the reference values $(1990,1994 a)$.

No correction for humidity is required if the calibration factor was referred to relative humidity of $50 \%$ and is used at a relative humidity between $20 \%$ and $80 \%$. If the calibration factor is referred to dry air a correction factor should be applied which is given in reference (1990).

In order to measure the machine room air pressure, we used a digital barometer name GTD 1100 manufactured by Greisinger Electronics of Germany. We also took temperature readings using the same instrument. Besides, for the measurement of phantom temperature we also placed a mercury thermometer placed within the water phantom for about 2 hours before starting the measurements for calibration and dosimetry. 


\subsubsection{Polarization correction factor $\left(K_{\mathrm{pol}}\right)$}

The effect of polarizing potentials, of opposite polarity, on an ion chamber must always be checked on commissioning and dosimetry. When a chamber is used in a beam that produces a measurable polarity effect, the true reading is taken to be the mean of absolute values of readings taken at both polarities. The polarization correction factor accounts for the differences in the collection of charges for the applied, positive or negative, potentials of $\pm \mathbf{3 0 0} \mathbf{V}$. Equation 3, given below, accounts for this correction factor.

$$
K_{P O L}=\frac{M_{+}+M_{-}}{2 M_{+}}
$$

Where, $\mathrm{M}_{+}$and $\mathrm{M}_{-}$are the electrometer readings, in coulombs, obtained with an applied polarity of voltage, positive and negative, respectively. For routine dosimetry checks like daily, weekly or monthly, it is sufficient to measure ' $\mathbf{M}$ ', the electrometer reading, with any polarity of voltage (i.e. positive or negative) but use that polarity subsequently in all dosimetry checks.

To be specific, for our objectives of commissioning, QA and treatment delivery we used all the measurements of correction factors, as outlined in equation 1. This is imperative to secure a precise calibration in order to obtain an absolute dose that is delivered by the machine. In our case, we used equation 3 , where

$\mathrm{M}_{+}=$Reading at voltage $+300 \mathrm{~V}$

$\mathrm{M}_{-}=$Reading at voltage $-300 \mathrm{~V}$

The reading $M_{+}$and $M_{-}$should be made with care, ensuring that the chamber reading is stable following any change in polarity (e.g. some chambers can take up to 20 minutes to stabilize). This may minimize the influence of fluctuations in the output of radiation generators.

\subsubsection{Ion Recombination factor $\left(K_{\mathrm{s}}\right)$}

The incomplete collection of charge in an ionization chamber cavity, due to the recombination of ions, requires the use of a correction factor $\mathbf{K}_{\mathbf{S}}$. Two separate effects take place in an ionization chamber viz. i) the recombination of ions formed by separate ionizing particle tracks, termed general (or volume) recombination, which is dependent on the density of ionizing particles and therefore on the dose rate, and ii) the recombination of ions formed by a single ionizing particle track, referred to as initial recombination which is independent of the dose rate. Both of these effects depend on the chamber geometry and on the applied polarizing voltage (1990). The Ion Recombination factor is given by,

$$
\mathrm{K}_{\mathrm{s}=} \frac{\left(\frac{v_{1}}{v_{2}}\right)^{2}-1}{\left(\frac{v_{1}}{v_{2}}\right)^{2}-\left(\frac{M_{1}}{M_{2}}\right)}\left[\mathrm{v}_{1 / \mathrm{V}_{2}}=2\right]--\cdot-\cdot-\cdot-\cdot-\cdot-\cdot-\cdot
$$

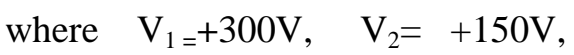

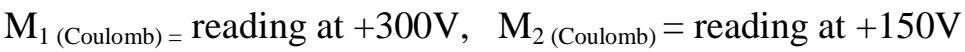

\subsubsection{Measurements and Results}

\section{a. Measurement of $\mathbf{K}_{\mathrm{TP}}$ (equation 2):}

Equipment used for this measurement has been mentioned earlier. The reference and the measured values are:

$\begin{array}{cc}\text { Reference Pressure: } 1013.25 \mathrm{hpa} & \text { Reference Temperature: } 20^{\circ} \mathrm{C} \\ \text { Measured Pressure: } 996.1 \mathrm{hpa} & \text { Measured Temperature: } 24^{\circ} \mathrm{C}\end{array}$ 
Therefore, Temperature and Pressure correction factor, $\mathbf{K}_{\mathrm{TP}}=\mathbf{1 . 0 3 1 1 0 4}$

b. Measurement of $\mathbf{K}_{\mathrm{S}}$ (equation 4 )

Measured data are tabulated in Table 11.

Table 11: Measurement data required to calculate the recombination of ions

\begin{tabular}{|l|l|}
\hline $\begin{array}{l}\text { Voltage , V, } \\
\text { (Volts) }\end{array}$ & $\begin{array}{l}\text { Meter Reading, Coulomb, } \\
(\mathbf{C})\end{array}$ \\
\hline+300 & $3.506 \times 10^{-8}$ \\
\hline+150 & $3.501 \times 10^{-8}$ \\
\hline-300 & $3.510 \times 10^{-8}$ \\
\hline
\end{tabular}

From above, the Ion correction factor, $\mathrm{K}_{\mathrm{S}}: 1.00044$---------------(equation4)

c. Polarity Correction factor, $\mathbf{K}_{\text {pol }}$

Our measured data produced this factor as $\mathrm{K}_{\mathrm{pol}}$ : 1.00052-------------(equation 3)

d. Dose calibration factor, $\mathbf{N}_{\mathrm{DW}}$

This calibration factor is given by IBA certificate. For our device, the value is:

$\mathrm{N}_{\mathrm{DW}}=4.77 \times 10^{7} \mathrm{~Gy} / \mathrm{C}$ (IBA chamber certificate)

e. Dose at $\mathbf{D}_{\mathbf{w}}$ : $246.22 \mathrm{cGy} / \mathrm{min}$ (equation 1)

PDD has been measured at a depth of $5 \mathrm{~cm}$ (which is $D_{w}$ in our case), and the maximum dose at 0.5 $\mathrm{cm}$ depth was determined by using equation 1, after incorporating all the correction factors.

Delta Hospital Ltd.

6/20/2013 1:29 PM Radiation Oncology Physics Division.

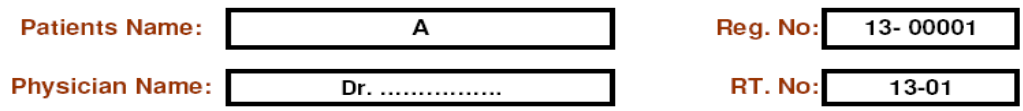
Machine Dose Rate: $246.22 \mathrm{cGy} / \mathrm{min}$

Physician Name: RT. No: Remarks New- Plan

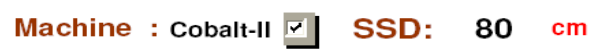

\begin{tabular}{||c|c|c|c|c|c||}
\hline \multicolumn{6}{||c||}{ New- Plan } \\
\hline \multicolumn{1}{||c|c||}{} & \multicolumn{5}{|c|}{ Remarks } \\
\hline al & Depth & $\begin{array}{c}\text { PDD } \\
(\%)\end{array}$ & $\begin{array}{c}\text { Machine } \\
\text { Output }\end{array}$ & $\begin{array}{c}\text { GD. } \\
\text { (cGy) }\end{array}$ & TT (min) \\
\hline 00 & 9.0 & 60.50 & 246.22 & 83 & 0.34 \\
\hline 00 & 8.0 & 64.70 & 246.22 & 77 & 0.31 \\
\hline 00 & 14.0 & 42.40 & 246.22 & 118 & 0.48 \\
\hline 00 & 14.0 & 42.40 & 246.22 & 118 & 0.48 \\
\hline
\end{tabular}
Shielding:

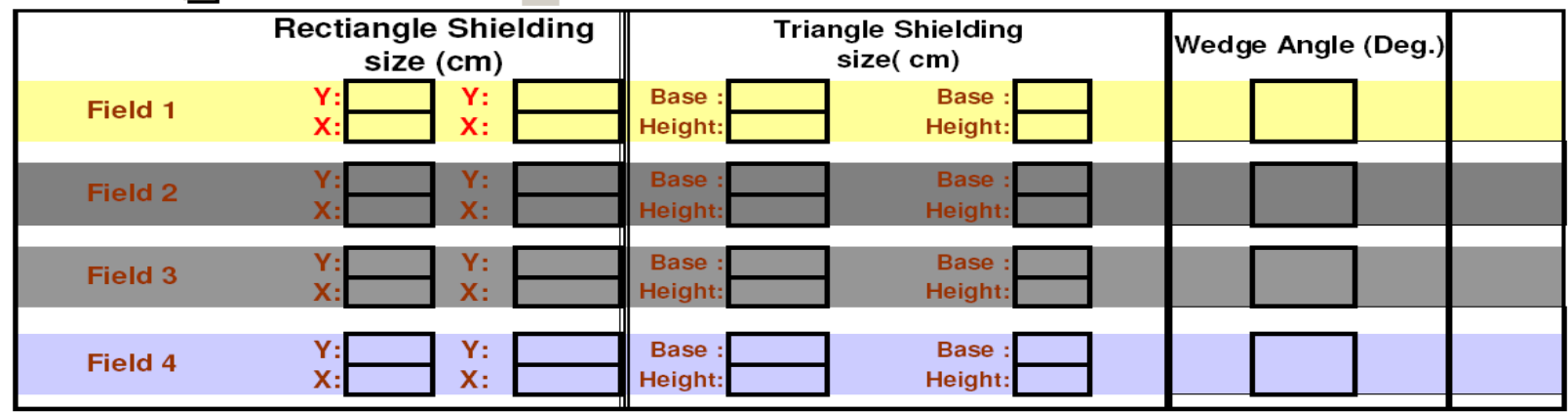

Physicist: Dr. Sadiq R. Malik

Check By:

Fig 9: A sample of Cobalt Machine Treatment Time (TT) Calculation Spread Sheet 
4.6 Cobalt-60 Treatment time calculation spreadsheet to deliver prescription dose delivery To calculate the patient treatment time (TT) manually, equation 5 below has been used. Use of equivalent square field size, standard $P D D$ and TMR values were from BJR25.

BJR Supplement 25, 1996 (Attix 1986, Khan 1984b) provides the 'TT' as,

$$
T T=\frac{\text { Pr escribe.Dose } @ \text { Tumor } \times 100}{(\text { PDD.or.TMR }) \times \text { Output }- \text { factor }} \text {................(5) }
$$

An in-house Excel Spreadsheet has been developed at Delta Hospital Ltd. to calculate the treatment Time to deliver the prescribed dose $\left(\mathrm{R}_{\mathrm{X}}\right)$ to the patient. This calculation sheet has been developed and used by us to determine the ' $\boldsymbol{T T}$ ' for each patient and for each field. The $T T$ is cross checked manually before delivery of the treatment to patients. A sample of this spreadsheet is given in Figure 9, followed by a brief description.

\section{A: Brief description of the Spread Sheet}

i. This Spread sheet can be used only for ${ }^{60} \mathrm{Co}$ at $S S D=80 \mathrm{~cm}$

ii. SSD or SAD of Treatment time calculation is done using this spread sheet

iii. Machine Dose rate will be changed every month by $1.09 \%$

iv. Equivalent Field size will be determined after inserting the $Y \& X$ field size values.

v. A Shielding block (e.g. rectangle, triangle or irregular shapes), if inserted, the equivalent square field size is calculated.

vi. Machine Output, Percentage Depth dose and Treatment time will be generated depending on the equivalent Square Field Size

vii. For a Given Dose a value of $\mathbf{0 . 5} \mathrm{cm}$ is typed in Depth Field

viii. Caution: This spread sheet dose rate is dependent on computer date \& time.

\subsection{Constancy Check (1994a)}

Constancy check refers to the repeated measurements at a fixed time of irradiation while collecting the charges as readings as tabulated in table 12. Procedure and set up for such a measurement is given below:

Measurement in water at SSD $=80 \mathrm{~cm}$, Field Size $=10 \times 10 \mathrm{~cm}^{2}$ at a measurement depth of $5 \mathrm{~cm}$ for a set time (t) $=\mathbf{1}$ minute. Number of readings are taken 10-15 times using an IBA FC-65 $\mathbf{P}$ chamber, Electrometer Dose-I and 1Dimensional water phantom

$$
\text { Standard Deviation or Constancy }(\sigma)=\frac{\sqrt{\sum(\bar{X}-X)^{2}}}{N}---------(6)
$$

Where, $\mathrm{N}=$ Number of Reading, $\mathrm{X}=$ Individual Reading, $\bar{X}=$ Average of all Readings.

The measurements for a time of one minute are tabulated in Table 12.

The differences in individual readings with that of the average value are small, almost negligible, when compared with that of the average value. Therefore, the deviation from the individual data implies constancy in the beam output and hence acceptable for patient treatment.

\subsection{Measurements of Output Factor}

The changes in the readings of the electrometer are tabulated in table 13. The data shows that there is a decrement and increment in such readings with the field sizes, smaller to bigger. The output factor is the ratio of readings in comparison to that of a standard field size, $10 \times 10 \mathrm{~cm}^{2}$.

For measurement of the output factor, we have used IBA 1-D Phantom, FC-65P chamber and IBA Electrometer Dose-I. Firstly, we setup the phantom at $80 \mathrm{~cm}$ (SSD, source to water surface distance) 
and placed the chamber at a depth of $5 \mathrm{~cm}$. The field size has been set up at $10 \times 10 \mathrm{~cm}^{2}$. Measured values are tabulated followed by calculating the output factor (OF) for different field sizes as given in table 13. The output factors for different field sizes, $4 \times 4$ to $32 \times 32$, are calculated with reference to the reading at 10x10field size.

\begin{tabular}{|c|c|c|c|c|}
\hline \multicolumn{5}{|c|}{ Table 12: Time vs. Deviation from the average value of the electrometer reading } \\
\hline $\begin{array}{l}\text { Time } \\
\text { (minute) }\end{array}$ & $\begin{array}{l}\text { Reading X } \\
\text { (Coulomb) } \\
\left(10^{-8}\right)\end{array}$ & $\begin{array}{l}\text { Average, } \bar{X} \\
(\text { Coulomb })\left(10^{-8}\right)\end{array}$ & $\begin{array}{c}\overline{\mathrm{X}}-\mathrm{X} \\
(\text { Coulomb })\left(10^{-8}\right)\end{array}$ & $\begin{array}{c}\text { Standard Deviation }(\boldsymbol{\sigma}) \\
(\text { Coulomb })\left(10^{-8}\right)\end{array}$ \\
\hline \multirow{10}{*}{1} & 3.506 & \multirow{10}{*}{3.5058} & -0.0002 & \multirow{10}{*}{0.0005} \\
\hline & 3.506 & & -0.0002 & \\
\hline & 3.506 & & -0.0002 & \\
\hline & 3.505 & & 0.0008 & \\
\hline & 3.505 & & 0.0008 & \\
\hline & 3.506 & & -0.0002 & \\
\hline & 3.507 & & -0.0012 & \\
\hline & 3.506 & & -0.0002 & \\
\hline & 3.506 & & -0.0002 & \\
\hline & 3.505 & & 0.0008 & \\
\hline
\end{tabular}

We have two Cobalt-60 radiotherapy machines (I, II).Our main goal is to see the variation of output with the field sizes. Table 13 also presents the Output Factors (OF) for the two machines and these results are plotted in Fig.10. This measurement is useful when one machine breaks down and patients are transferred to the other for treatments. As can be seen, the differences of respective output factors between the two machines are very small, almost negligible and hence compatible to each other. Fig. 10 also shows that the $\boldsymbol{O F}$ increases with the increase of the field size.

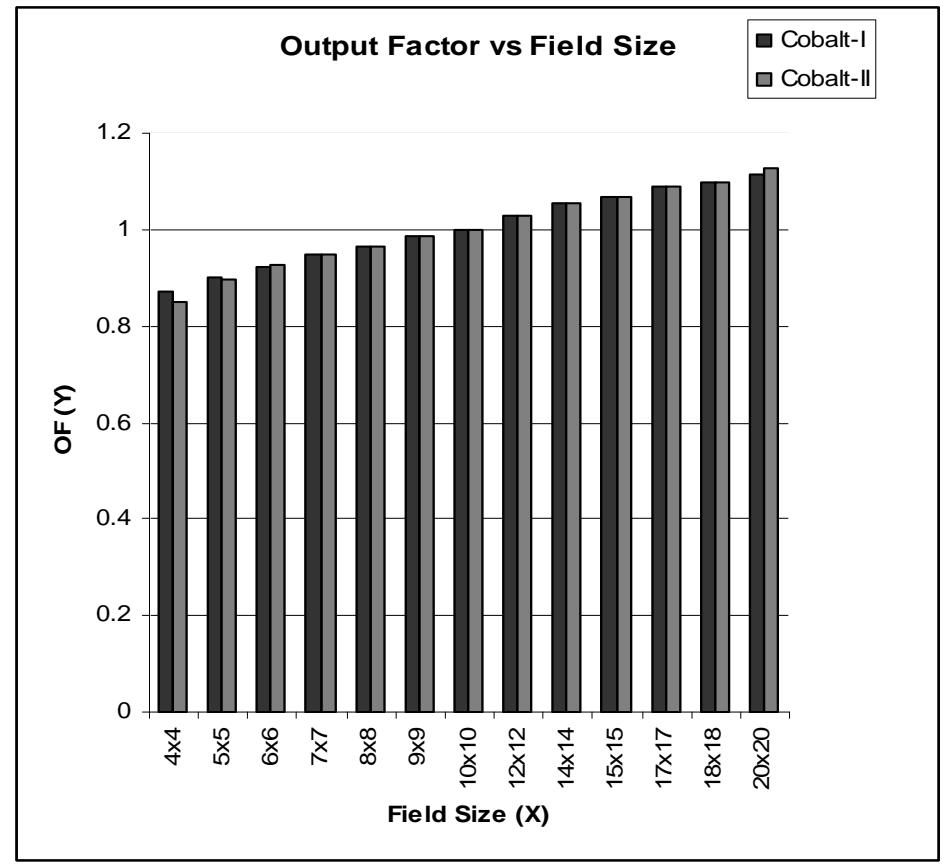

Fig 10: Field size vs. output factor for two Cobalt Machines. 
Table 13: Field size vs. Output factor $(\mathrm{OF})$

\begin{tabular}{|l|l|l|}
\hline $\begin{array}{l}\text { Field Size } \\
(\mathbf{c m})\end{array}$ & $\begin{array}{c}|c| \\
\text { 'OF } \\
\text { Cobalt-1 }\end{array}$ & $\begin{array}{l}\text { 'OF' } \\
\text { Cobalt-II }\end{array}$ \\
\hline $4 \times 4$ & 0.8703 & 0.8491 \\
\hline $5 \times 5$ & 0.9007 & 0.8960 \\
\hline $6 \times 6$ & 0.9235 & 0.9266 \\
\hline $7 \times 7$ & 0.9473 & 0.9465 \\
\hline $8 \times 8$ & 0.9665 & 0.9665 \\
\hline $9 \times 9$ & 0.9856 & 0.9852 \\
\hline $10 \times 10$ & 1.0000 & 1.0000 \\
\hline $12 \times 12$ & 1.0299 & 1.0310 \\
\hline $14 \times 14$ & 1.0558 & 1.0548 \\
\hline $15 \times 15$ & 1.0665 & 1.0676 \\
\hline $17 \times 17$ & 1.0888 & 1.0888 \\
\hline $18 \times 18$ & 1.0976 & 1.0972 \\
\hline $20 \times 20$ & 1.1146 & 1.1268 \\
\hline $22 \times 22$ & 1.1305 & 1.1300 \\
\hline $23 \times 23$ & 1.1360 & 1.1364 \\
\hline $25 \times 25$ & 1.1476 & 1.1465 \\
\hline $27 \times 27$ & 1.1563 & 1.1556 \\
\hline $28 \times 28$ & 1.1608 & 1.1589 \\
\hline $30 \times 30$ & 1.1667 & 1.1657 \\
\hline $32 \times 32$ & 1.1707 & 1.1697 \\
\hline & & \\
\hline
\end{tabular}

4.9 Dose Variation Evaluation with Daily QA-3 Equipment

This device has 12 Diodes to checkup the field size and 13 Ion Chambers to evaluate the central axis dose and elsewhere as illustrated in Fig. 11.

All of the above mechanical and dosimetry measurements have been completed to substantiate and confirm the acceptance, commissioning and treatment delivery for such a teletherapy machine (Sun). . The dose calibration has been carried out previously and described in above mentioned measurements. Another measurement of dose delivery has been introduced by us using a new device called QA-3 to show the central dose for the Co-60 source. Calibration for this device has been done by us to evaluate the dose at Central Axis $(\boldsymbol{C A X})$ for an SSD of $80 \mathrm{~cm}$ and for a field size of $20 \times 20 \mathrm{~cm}$. Here, we use a field size of $20 \times 20 \mathrm{~cm}$. In the device QA-3, diodes are positioned, by the manufacturer, at 20x20 cm area of Daily QA -3. Fig 11 shows the dose at various points of the device. This device is made by SUN NUCLEAR corp., USA.

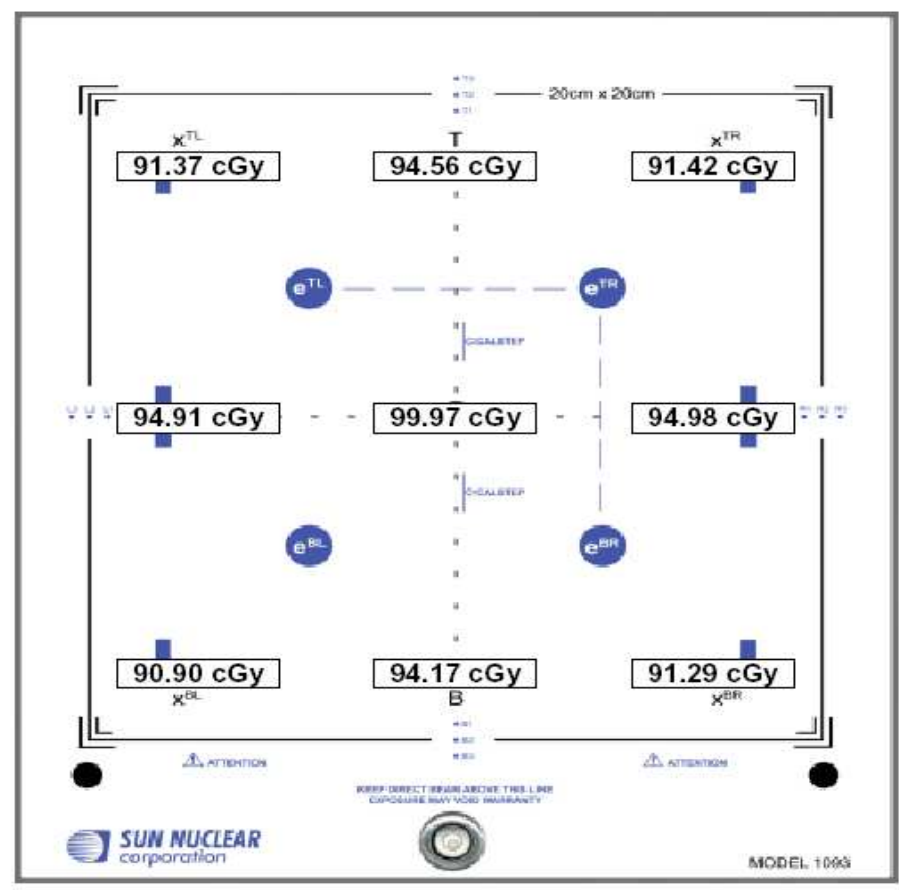

Fig 11: Results are shown here by using the Daily QA-3 of SUN NUCLEAR Device SN: 81374052.The central Axis (CAX) is expected to be $100 \mathrm{cGy}$. 


\subsection{DISCUSSION}

Machine parameters are verified and elaborate measurements have been carried out to ascertain the consistent functioning of the machine with the manufacturer's specifications. Measurements reported here are systematically and sequentially presented in this tutorial to commission a teletherapy machine. All of the necessary procedures are required to be followed to acquire accurate values for the delivery of exact dose. The treatment machine will then be certified and commissioned for the treatment of Cancer Patients. Incidentally, it may be mentioned here that there are about 12 Cobalt Teletherapy Units alone existing in Bangladesh now and more to be installed in the near future (2013). Clinical data have been acquired and used here, which relate to the standard protocols and tolerance values of the machine parameters. It is demonstrated here that all of the above work is needed for a precise dose delivery to tumors. It is, therefore, recommended to carry out the measurements, step by step, to accept the teletherapy machine for treatments. Treatment quality and delivery are imperative in the reliable use of the ${ }^{60} \mathrm{Co}$ Teletherapy Machine in any Cancer Center.

\section{Acknowledgment}

Our sincere thanks are due to Delta Hospital Ltd. and the Managing Director, Professor S. Mukarram Ali, FRC Path, in providing the UNITS and encouragement respectively to work on ${ }^{60} \mathrm{Co}$ machines described here. Thanks are also due to Professor M. N. Huda, M.D. the Principal Oncologist and Dr. Parvin A. Banu, M.D., Chief Oncologist and Dr. (Lt.Col.) M. S. Sarwar Alam, DMRT, FCPS, Head of the Oncology Department, for their support in the clinical use of the Teletherapy Units, for cancer treatments.

\subsection{Bibliography}

1990. AAPM, s TG-51 protocol for clinical reference dosimetry of energy photon and electron beams. Medical Physics, 26. 1847-1819.

1994a. AAPM Report No. 13: Physical Aspect of quality Assurance in radiation therapy. American Association of Physicists in Medicine.

1994b. Task Group 40: Comprehensive QA for Radiation Oncology: Report of AAPM Radiation Therapy Committee. Med. Phys., 21. 581.

2004. Acceptance/Quality Assurance tests for Medical linear Accelerator. Medical Physics \& safety section, Radiological Physics \& Advisory Division, Bhaba Atomic Research Center.

2005. IAEA TRS-398: Absorbed dose determination in external beam radiotherapy. An International code of practice for dosimeter.

2013. RE: Private communication: Dr. Emdadul Haque, Director, Bangladesh Atomic Energy Commission (BAEC)

Allen BJ \& Blagojevic N. 1996. Alpha and beta emitting radiolanthanides in targeted cancer therapy: the potential role of terbium-149. Nucl Med Communications. 40-47.

Attix FH 1986. Atmospheric Corrections. Introduction to Radiological Physics and Radiation Dosimetry. 327. N.Y., USA, John Wiley and Sons.

Cunningham J, et al. 1983. The Physics of Radiology. Thomas CC, IL., Springfield.

Khan Faiz 1984a. The Physics of Radiation Therapy. Chapter 17.

Khan Faiz 1984b. The Physics of Radiation Therapy: A-14.

Metcalfe Peter \& Kron Thomas 1997. The Physics of Radiotherapy X-Rays from Linear Accelerators. Medical Physics Publishing.

Sun. Sun Nuclear Corporation, Device: Daily $Q A$ 3, P/N: 1093000-0 [Online]. www.sunnuclear.com. 